\title{
Statistical Method to Detect Subsurface Objects Using Array Ground-Penetrating Radar Data
}

\author{
Xiaoyin Xu, Eric L. Miller, Member, IEEE, Carey M. Rappaport, Senior Member, IEEE, and Gary D. Sower
}

\begin{abstract}
We introduce a combination of high-dimensional analysis of variance (HANOVA) and sequential probability ratio test (SPRT) to detect buried objects from an array ground-penetrating radar (GPR) surveying a region of interest in a progressive manner. Using HANOVA, we exploit the transient characteristic of GPR signals in the time domain to extract information about buried objects at fixed positions of the array. Based on the output of the HANOVA, the SPRT is employed to make detection decisions recursively as the array moves downtrack. The method is on-line implementable and of low computational complexity. Our approach is validated using field-data from two quite different GPR sensing systems designed for landmine detection applications.
\end{abstract}

Index Terms-Analysis of variance (ANOVA), array signal processing, GPR mine detection, sequential detection, transient signal analysis.

\section{INTRODUCTION}

G ROUND-PENETRATING radar (GPR) is widely used in detecting subsurface objects such as buried landmines, unexploded ordnance, and utility lines [1]. Compared with other subsurface sensing technologies, GPR has a few advantages. First, it is sensitive to changes in all three electromagnetic characteristics of a medium, electric permittivity, electric conductivity, and magnetic permeability. Thus GPR is capable of detecting both metallic and nonmetallic objects. Second, unlike sensors that can only survey an area directly beneath them, GPR can survey an area in front of it [2], [3]. Therefore, a GPR system can be used to detect dangerous objects before the system moves over and past them. This can be important for operations such as buried landmine detection and unexploded ordinance remediation. In practice, GPR data are either processed on-line or off-line. On-line processing can be fast and provides real-time detection and requires a more sophisticated hardware and powerful computer [4]. Off-line processing requires a large amount of data storage and can use data of a full scan [5].

A typical GPR transmitter/receiver configuration is shown in Fig. 1(a). The system consists of one transmitter and one receiver. The transmitter emits a short pulse of electromagnetic energy and the receiver collects the echo for a certain time period. The exact type of the transmitter and receiver, shape of the electromagnetic pulse, and system setup depend on the specific

Manuscript received May 2, 2001; revised December 28, 2001. This work was supported by an ARO MURI on Demining under Grant DAAG55-97-1-0013.

X. Xu, E. L. Miller, and C. M. Rappaport are with the Center for Subsurface Sensing and Imaging Systems, Department of Electrical and Computer Engineering, Northeastern University, Boston, MA 02115 USA (e-mail: elmiller@ece.neu.edu).

G. D. Sower is with the EG\&G MSI, Inc., Albuquerque, NM 87106 USA.

Publisher Item Identifier S 0196-2892(02)04622-3. application of the GPR [1], [6]-[8]. To improve performance and efficiency, a GPR array is usually employed to sweep a large area in a relatively short time. Fig. 1(b) shows a typical GPR array moving in the $x$ direction. At every stop of the array, the GPR array operates in the following sequence: 1) the first transmitter radiates a pulse into the ground and then turns off; 2) the first receiver turns on to collect reflected signal; and 3) the first receiver turns off after a short time, usually 10 to $20 \mathrm{~ns}$. The above process repeats from every pair of transmitters and receivers and then the GPR array moves to next position. Based on the echoes, the processing objective is to determine if an object is present in the GPRs field of view.

The inherent near-field nature of the GPR detection problem coupled with the fact that the objects of interest are embedded in an inhomogeneous halfspace with a typically rough interface present some significant challenges in the area of GPR signal processing. Indeed, assuming one has detailed knowledge of the air-earth interface as well as the electrical properties of the subsurface, just modeling the received signal using, for example, a three-dimensional (3-D) finite difference time-domain code, is a daunting task [6], [9]. The use of such a forward model in any form of on-line processing routine where one might need to account for, e.g., unknown ground structure is clearly infeasible at the current time.

Thus, here we consider detection methods which are less computationally demanding with an eye toward approaches that could be used in real-world scenarios. Our interests are in techniques possessing three important characteristics. First, to reflect the manner in which GPR data are acquired and the nature of the GPR mission, the algorithms should be causal in that they need only the data at the current and previous sensor position to determine whether an object is present in the field of view of the sensor. Second, they should be of low complexity. Preferably the number of calculations would grow linearly with the size of the data set. Finally, the processing schemes should be robust to uncertainties in the GPR environment and hence the particular detailed structure of the received signals.

Current signal processing methods with some or all of these characteristics fall into one of three categories. First, pattern matching methods [10] employ techniques such as fuzzy set theory and neural networks. Such methods can be fast but also require extensive training to function well. Second, image-thendetect techniques [11] employ a beamforming or backpropagation approach to build an image of the subsurface which is then post-processed to detect objects. These approaches generally require the data from the full GPR scan to form an image and are thus not well suited to on-line computations in which information is processed sequentially as the array proceeds down-track. 


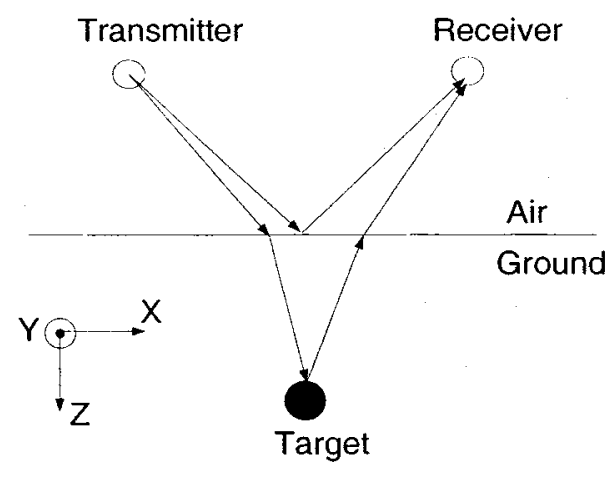

(a)

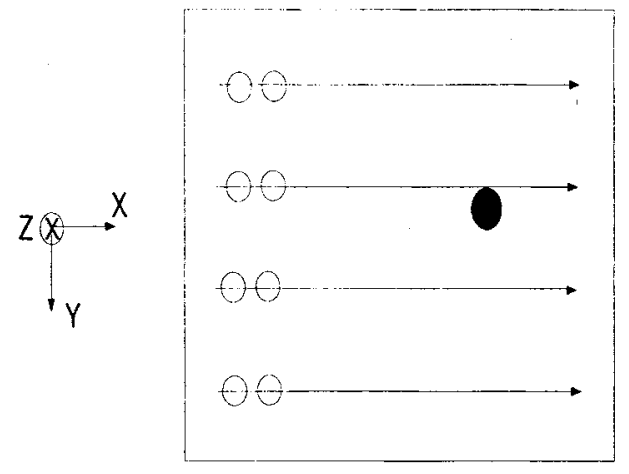

(b)

Fig. 1. Setup: (a) a single GPR system and (b) a GPR array (plane view).

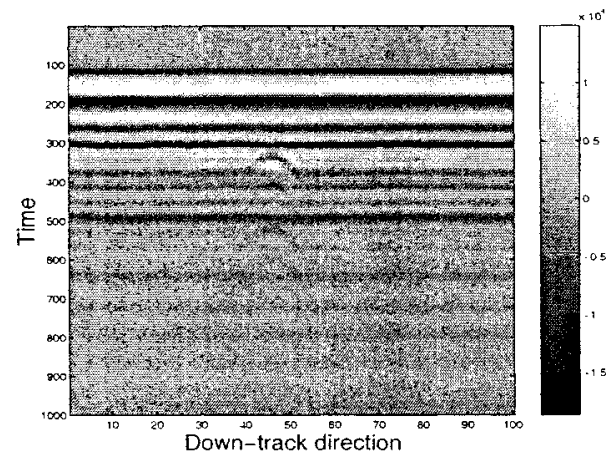

(a)

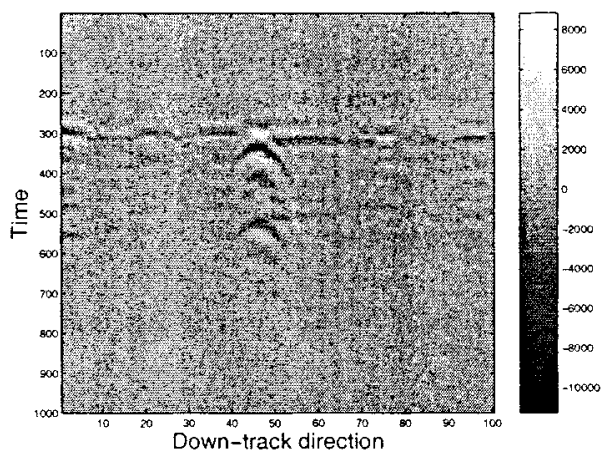

(b)

Fig. 2. Observation from one T/R pair, for a metal mine TM15 buried at position 45. (a) Raw data. (b) Data after mean subtracted. Unit in down-track position is about $7.6 \mathrm{~cm}$. Unit in time axes is $0.02 \mathrm{~ns}$.

Finally, there has been much work done in statistical signal processing, where one can employ statistical tools to detect objects and examine quantities such as probability of detection and probability of false-alarm [12].

Here we consider a statistical, transient detection approach. By "transient" we mean that the signals of interest are manifest in the GPR data for a small number of sensor positions and for relatively few samples in any received waveform. For example, in Fig. 2 we plot raw observations obtained by one transmitter/receiver (T/R) pair from an EG\&G GPR system [13], over an M20 metal mine. Each column of this image is a time-series of observations for a given stop of the array. It is seen that the received GPR signal is transient in two ways. First, for each time-series (i.e., for each column of the image) containing an object signal, the signal appears only in a brief window, roughly from samples 350 to 700 . The reason is that the object signal always comes after the signal arising from the bounce off of the air-ground interface and attenuates quickly in lossy media. Second, the object signal shows up only at a few down-track positions of the GPR array, specifically locations 50 through 65 . In both cases, the appearance of object signal changes the mean value of the data. Our method for object detection then is based on detecting change in this mean first in the cross-track direction and then in the down-track direction.
More specifically, our approach consists of two parts. First, at each down-track position of the array, we process the data among all T/R pairs to generate one test statistic. We use high-dimensional analysis of variance (HANOVA) [14] to test whether the data consists of reflected signal from a buried object. The HANOVA is a generalized version of standard analysis of variance (ANOVA) [14], [15], which is a method for testing hypothesis about means of random vectors. Second, a sequential probability ratio test (SPRT) [4] is applied to process the statistic of the HANOVA as the array moves down-track. The SPRT is a recursive statistical hypothesis testing technique that provides early indication of the onset of changes in a time series. The output of the SPRT is compared with a threshold. If it exceeds the threshold, a detection is declared, otherwise, the GPR array moves one more step down-track and new data are collected and processed in the above manner.

As explained in greater detail below, our approach does in fact satisfy the three requirements we discussed previously. It is causal and has computational complexity that grows linearly with the size of the data. Moreover, we show through real-data examples that it is robust, requiring little in the way of training and able to successfully address the object detection problem for a number of GPR systems operating in a wide range of environments, such as Socorro test site in New Mexico and Dedham test site of Northeastern University in Massachusetts. 
We do stress here that the algorithm in this paper is intended only to find anomalies beneath the GPR array and not to solve the far more challenging classification problem. Thus, from a practical perspective our approach will serve well as an efficient "pre-screener" in a larger automatic target detection algorithm suite. Finally, our method is motivated by landmine detection using GPR, however it can also be used in other detection application, such as laser-induced acoustic subsurface objects detection [16].

The paper is organized as follows. Section II discusses the problem formulation and our method. Section III gives some examples of using the method in different situations. Field data from different radar configurations and test sites are used to show how the algorithm works. Conclusion and direction of future work are given in Section IV.

\section{PROBlem Formulation AND Algorithm}

To begin, we consider a single GPR T/R pairs as shown in Fig. 1(a). After each transmission, the receiver collects an echo for a certain amount of time. Depending on the presence of an object, there are either two or three components in the echo. One is measurement noise, assumed to be white and Gaussian. Another is background, i.e., "nominal" signal observed in object-free regions. The third component is object signal, reflection from a buried object.

For the GPR array shown in Fig. 1(b), assume we have $M$ GPR T/R pairs surveying an area in $N$ steps, the task is to use present and previous array measurement to detect buried mines as the array moves down-track. At each down-track position, we model the array detection problem in a typical hypotheses testing framework [4]

$$
\begin{aligned}
& H_{0}: \text { there is no object } \\
& H_{1}: \text { there is an object. }
\end{aligned}
$$

The null hypothesis $H_{0}$ means that there is no buried object in the field of view of the GPR array, so the total received signal is comprised of nominal background and measurement noise. By nominal background, we mean any portion of the received waveform not sensor noise and not arising from the interaction of the transmitted pulse with the object. Reflection from the air-ground interface is the dominant component of this part of the signal. The alternative hypothesis $H_{1}$ indicates that there is a buried object so that the received signal consists of nominal background, measurement noise, and an object signal.

In this paper, we assume that the nominal background signal has been removed via a preprocessing stage. The most often used background removal methods include casual methods, such as subtraction of a moving average from the observation [17], [18], and noncausal methods, such as subtraction of an ensemble average from the observation [19], [20]. Causal methods use data from previous and present collection, noncausal methods use data from previous and future collection. Other background removal methods include linear prediction method [21] and adaptive method [22]. In this paper, a moving average (MA) filter is used to eliminate the nominal background.
In practice, the receiver collects time samples of the reflection and stores it as a vector. For convenience, we use vector notation in our discussion, i.e., $\mathbf{y}(m, n)$ is a column vector representing observation of the $m$ th T/R pair at the $n$th down-track position. The length of $\mathbf{y}(m, n)$ is $K$, the number of samples in time. Fig. 3 shows the received signal after the nominal background removal. ${ }^{1}$ We then have the hypothesis test

$$
\begin{aligned}
& H_{0}: \mathbf{y}(m, n)=\mathbf{v}(m, n) \\
& H_{1}: \mathbf{y}(m, n)=\mathbf{s}(m, n)+\mathbf{v}(m, n)
\end{aligned}
$$

where $m=1, \ldots, M, n=1, \ldots, N$ are positions of GPR, $\mathbf{s}(m, n)$ is the assumed signal due to presence of a buried object, $\mathbf{v}(m, n)$ is assumed to be a white Gaussian noise with a zero mean, and covariance matrix $\sigma_{v}^{2} \mathbf{I}$, where $\mathbf{I}$ is the identity matrix of size $K$ and independent of $(m, n)$.

The statistical assumptions about $\mathbf{v}$ are not strictly accurate in describing the noise in a GPR signal. For example, the background removal process will not be perfect, leaving a component of correlated "clutter" in the data which may or may not possess Gaussian statistics. Despite the mismatch, the use of the additive white Gaussian noise (AWGN) model is useful for a number of reasons. This model allows us to develop an algorithm for object detection which is firmly rooted in Gaussian-based statistical decision theory and which can be generalized in the future for more complex noise processes. Moreover, the complexity of such algorithms is quite low making them well suited for real-world implementation. Finally, test results in Section III from real field data demonstrate that the method is quite effective in detecting objects. Thus, the Gaussian noise model is shown to work in practice. While it may be interesting to explore other, more accurate models for the sensor noise to determine for example what can be gained in terms of performance and what would be lost in terms of computational complexity, such an effort is beyond the scope of the work in this paper.

Based on the previous discussion, after background removal the hypothesis test in (1) may be written as

$$
\begin{aligned}
& H_{0}: \mathbf{y}(m, n) \sim N\left(\mathbf{0}, \sigma_{v}^{2} \mathbf{I}\right) \\
& H_{1}: \mathbf{y}(m, n) \sim N\left(\mathbf{s}(m, n), \sigma_{v}^{2} \mathbf{I}\right), \\
& \quad m=1, \ldots, M, n=1, \ldots, N
\end{aligned}
$$

where the notation $\mathbf{y} \sim N(\overline{\mathbf{x}}, \mathbf{R})$ indicates that $\mathbf{y}$ is distributed as a Gaussian random vector with mean $\overline{\mathrm{x}}$ and covariance matrix R.

As stated in the Introduction, we take a two-step approach to the processing of $\mathbf{y}(m, n)$. First, for each $n$ we use the HANOVA procedure to generate a single test statistic, $Y(n)$, from the data from all T/R pairs. Second, a recursive, sequential detection scheme is employed to process $Y(n)$ as we proceed down track in order to determine where objects are present.

\section{A. Cross-Track Processing}

We begin by discussing the use of HANOVA to process data in the cross-track direction. HANOVA is a generalized version

\footnotetext{
${ }^{1}$ For the purpose of illustration, in this section we use field data from a buried metal mine to illustrate clearly the concept under consideration. Examples which demonstrate better the utility of our approach on more challenging problems, including buried plastic mines, are given in Section III.
} 


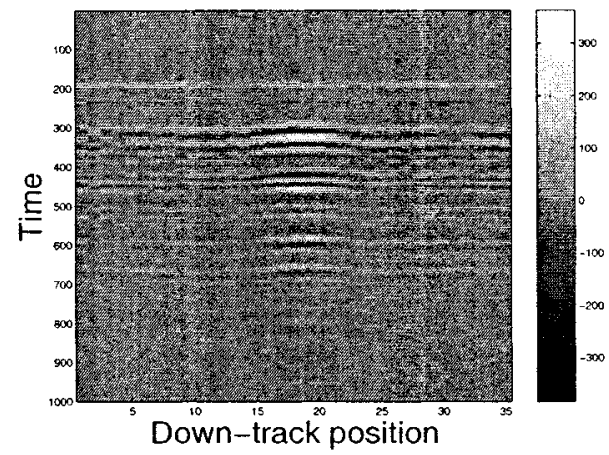

(a)

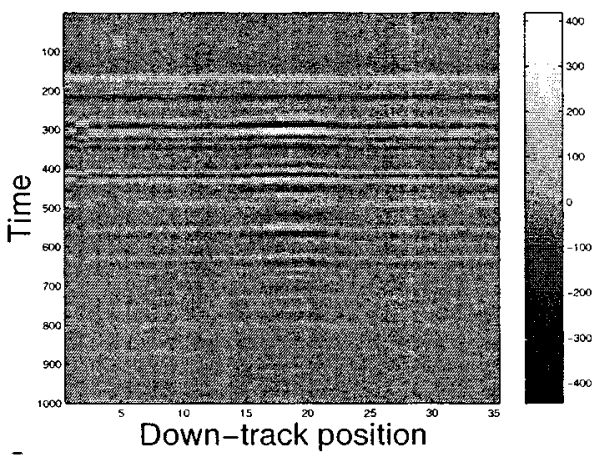

(c)

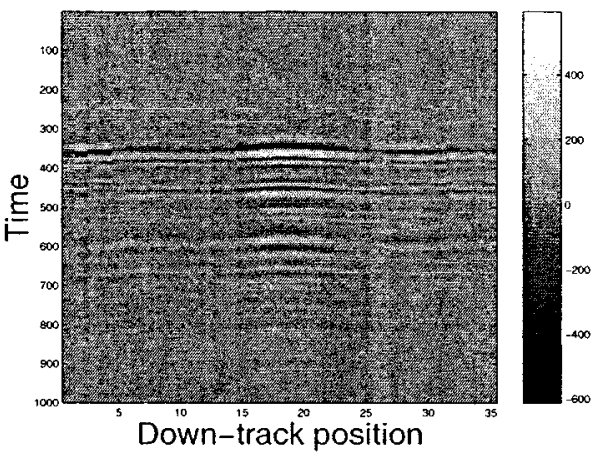

(b)

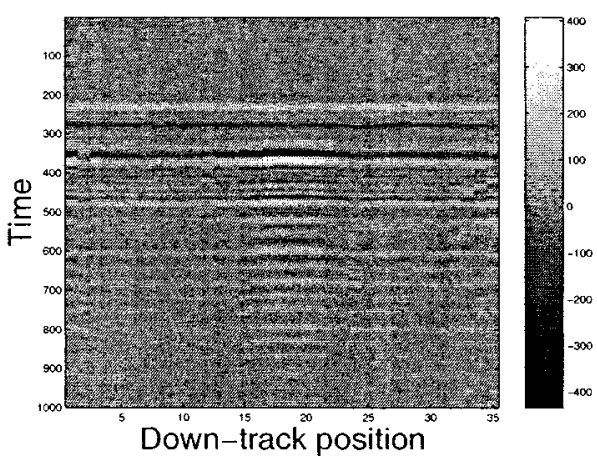

(d)

Fig. 3. Signals from four T/R pairs, after background removal. (a) Pair 1. (b) Pair 2. (c) Pair 3. (d) Pair 4. Unit in down-track position is about $7.6 \mathrm{~cm}$. Unit in time axes is $0.02 \mathrm{~ns}$.

of analysis of variance (ANOVA). ANOVA is a body of methods to analyze the data with a view to test hypotheses about the effects of one or more factors [23]. To review the basics of ANOVA, we follow the notation established above for the GPR problem and for simplicity assume we have one data vector of size $K \times 1$ from a single T/R pair with $\sigma_{v}^{2}$ normalized to one. Hence, $\mathbf{y} \sim N(\mathbf{s}, \mathbf{I})$ and we wish to test $H_{0}: \mathbf{s}=\mathbf{0}$ (i.e., no object) versus $H_{1}: \mathbf{s} \neq \mathbf{0}$ (i.e., an object present). ${ }^{2}$ Standard ANOVA is essentially an "energy detection" scheme [14] where we estimate $\mathbf{s}$ by $\mathbf{y}$, generate the test statistic $Y=\|\mathbf{y}\|^{2}$, and compare $Y$ to a threshold, $\gamma$. If $Y$ exceeds the threshold, $H_{1}$ is chosen, else $H_{0}$ is selected. The probability of detection of the standard ANOVA is [14]

$$
P_{d}\left(H_{1} \mid H_{1}\right)=Q\left(\frac{\gamma-\frac{\|\mathbf{s}\|^{2}}{\sqrt{2 K}}}{\sqrt{1+\frac{2\|\mathbf{s}\|^{2}}{K}}}\right)
$$

where $\gamma$ is the test threshold decided by setting an acceptable probability of false alarm under $H_{0}$ and $Q$ is the complementary cumulative distribution function (cdf) and is strictly decreasing [24]

$$
Q(Y)=\int_{\gamma}^{\infty} P_{1}(Y) d Y
$$

${ }^{2}$ For notional simplicity, we drop the explicit dependence of all quantities on $m$ and $n$ in this discussion.
Recently, Fan [14] and Fan and Lin [15] have noted that the performance of ANOVA suffers for problems when the signal of interest is limited to a window of the observation vector. The reason is that a full dimensional test loses its power due to accumulation of stochastic noise. To see why, suppose $\mathbf{s}$ is different from 0 only for say the first $k_{0}$ samples of the full observation vector. Then, on average, as $K>k_{0}$ goes large, $\sum_{k=1}^{K}[\mathbf{s}]_{k}^{2} / \sqrt{2 K}$ decreases due to the accumulation of zero mean noise samples and the term within the parenthesis of (3) increases, thus reducing $P_{d}$. Therefore, for higher probability of detection, we would like to confine the test on a window mostly containing the signal of the observation vector. The window we choose is a box window $\mathbf{w}$, defined as

$$
[\mathbf{w}]_{k}= \begin{cases}1, & k=k_{1}, \ldots, k_{2} \\ 0, & \text { otherwise }\end{cases}
$$

where $1 \leq k_{1}<k_{2} \leq K$. The $k_{1}$ and $k_{2}$ are chosen in a preset manner, as discussed later in this section. Multiplying each element in $y$ by the corresponding element of $\mathbf{w}$ gives the windowed $\mathbf{y}_{\mathbf{w}}$

$$
\left[\mathbf{y}_{\mathrm{w}}\right]_{k}=[\mathbf{y}]_{k} \times[\mathbf{w}]_{k}, \quad k=1, \ldots, K .
$$

This kind of method of reducing a full dimensional test to a windowed version is called HANOVA [15]. To demonstrate the utility of HANOVA, we test the time series shown in Fig. 4(a). We choose to test the vector at its full dimension $k_{1}$ in (5) is 1 and $k_{2}=1000$, and two windowed subdimensions (each 


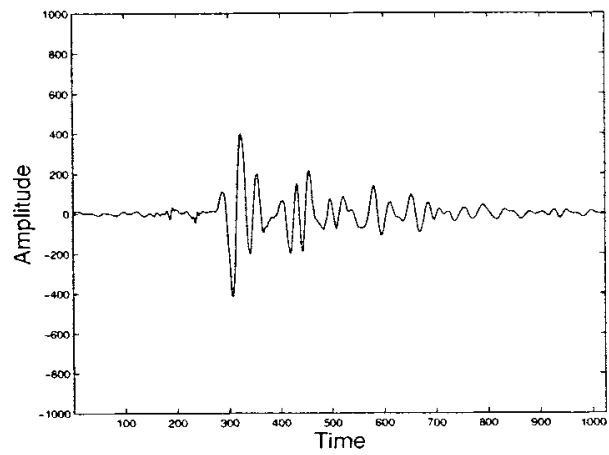

(a) Example signal collected when sensor over object, unit in time is $0.02 \mathrm{~ns}$.

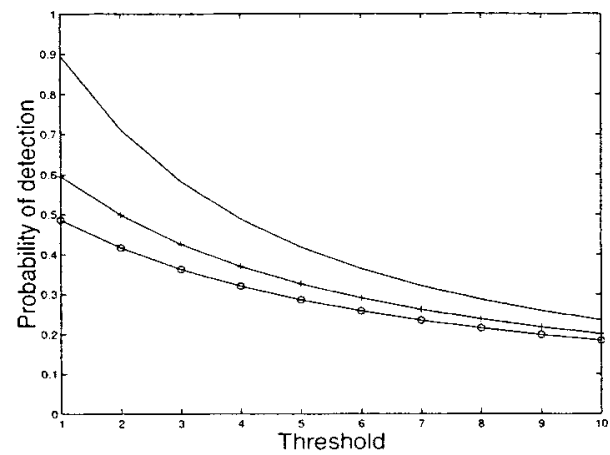

(b) Probabilities of detection vs. thresholds, solid line, a window from 200 through 800 , '- + ' line, a window from 100 to 900 , '-o' line, no window.

Fig. 4. Motivating example for use of HANOVA rather than ANOVA. (a) Sample data collected by a GPR when over an object. The transient nature of the relevant portion of the signal is clear. Using this signal, in (b) we display the decreasing detection rates associated with including increasing numbers of "noise" samples in the processing.

containing fewer and fewer noise components) $k_{1}=100$ and $k_{2}=900$, and $k_{1}=200$ and $k_{2}=800$. From Fig. 4(b), it is seen that, by setting the window properly, higher probability of detection is gained at different levels of detection thresholds, $\gamma$ in (3). It demonstrates that when signal is not "full-dimensional," looking for a window of signal-rich subdimensions to test will increase the probability of detection.

When the observation is a sequence of high-dimensional vectors whose components are mostly noise, as is the case for our GPR problem, it is desirable to adaptively choose the window to maximize the probability of detection. Fan's original work was limited to problems in which the first $k_{0}$ dimensions are believed to be signal-rich and used in HANOVA, with $k_{0}$ found from the data. Here we consider a generalization of Fan's work to take into account the fact that for the GPR problem the transient signal is significant over a window not generally starting with the first dimension but in the middle of the observation vector. Moreover, this window will vary with $(m, n)$.

To choose this window we note that (3) indicates that the probability of detection achieves its maximum value when the term inside the parenthesis is minimized. Fan [14] proposed a one-side maximization when there is prior information that large absolute values of $s$ are mainly located on the first $k$ dimensions. In his work, the window is chosen by finding a $k$ such that $[14]$

$$
\underset{k: 1 \leq k \leq K}{\arg \max }=\frac{\sum_{i=1}^{k}[\mathbf{y}]_{i}^{2}}{\sqrt{k}}-\sqrt{k} .
$$

Based on Fan's work, we generalize it to a two-side maximization, in which we want to maximize the quantity

$$
\underset{k_{1}, k_{2}}{\arg \max } \frac{\sum_{k=k_{1}}^{k_{2}}[\hat{\mathbf{y}}(m, n)]_{k}^{2}}{\sigma_{v}^{2} \sqrt{k_{2}-k_{1}+1}}-\sqrt{k_{2}-k_{1}+1}
$$

where $1 \leq k_{1}<k_{2} \leq K, \sigma_{v}$ is the standard deviation defined in (2), and $\hat{\boldsymbol{y}}$ is the mean value of the previous $l$ vectors, defined as

$$
\hat{\mathbf{y}}(m, n)= \begin{cases}\frac{\sum_{j=1}^{n} \mathbf{y}(m, j)}{j}, & n=1, \ldots, l \\ \frac{\sum_{j=n-l}^{n} \mathbf{y}(m, j)}{l}, & n>l\end{cases}
$$

where $m=1, \ldots, M$ and the corresponding window $\mathbf{w}(m, n)$ is decided based on $\hat{\mathbf{y}}(m, n)$ as $k_{1}, k_{2}$ are defined by (7). More will be said about choosing a proper $l$ in the Appendix.

Rather than looking for the optimal window by searching over all $\left\{k_{1}, k_{2}\right\}$ pairs, we pursue a suboptimal, but more efficient two-stage approach. First, we fix $k_{1}$ as 1 , incrementally increase $k_{2}$, and stop when (7) is maximized. Thus, we determine the end point of the window $k_{2}$. Starting from $k_{2}$, working backward toward the first point, we similarly determine the starting point of the window, $k_{1}$. In the backward search, to make the mathematics concise, we introduce a new operator $\Phi$, which takes a vector as its input and rearrange the elements backward, i.e.,

$$
\Phi\left(\left[y_{1}, y_{2}, \ldots, y_{n-1}, y_{n}\right]^{T}\right)=\left[y_{n}, y_{n-1}, \ldots, y_{2}, y_{1}\right]^{T}
$$

where the superscript $T$ denotes transpose. Both searching steps can be computed in linear complexity: it takes $o(K)$ steps to find the $k_{2}$ and $o\left(k_{2}\right)$ steps to find the $k_{1} .{ }^{3}$ In summary, the steps for looking for windows at the $n$th stop of the GPR array are given in Fig. 5.

Having determined the window at the position $(m, n)$, the next stage of processing is to generate a single detection statistic at stop $n$. Here we generalize HANOVA to multiple vector observations via

$$
Y(n)=\frac{1}{\sigma_{v}^{2}} \sum_{m=1}^{M}\left\|\mathbf{y}_{\mathrm{w}}(m, n)\right\|^{2} .
$$

\footnotetext{
${ }^{3}$ The notation $o(K)$ means that the computational complexity grows slower than or equally fast as $K$ increases.
} 


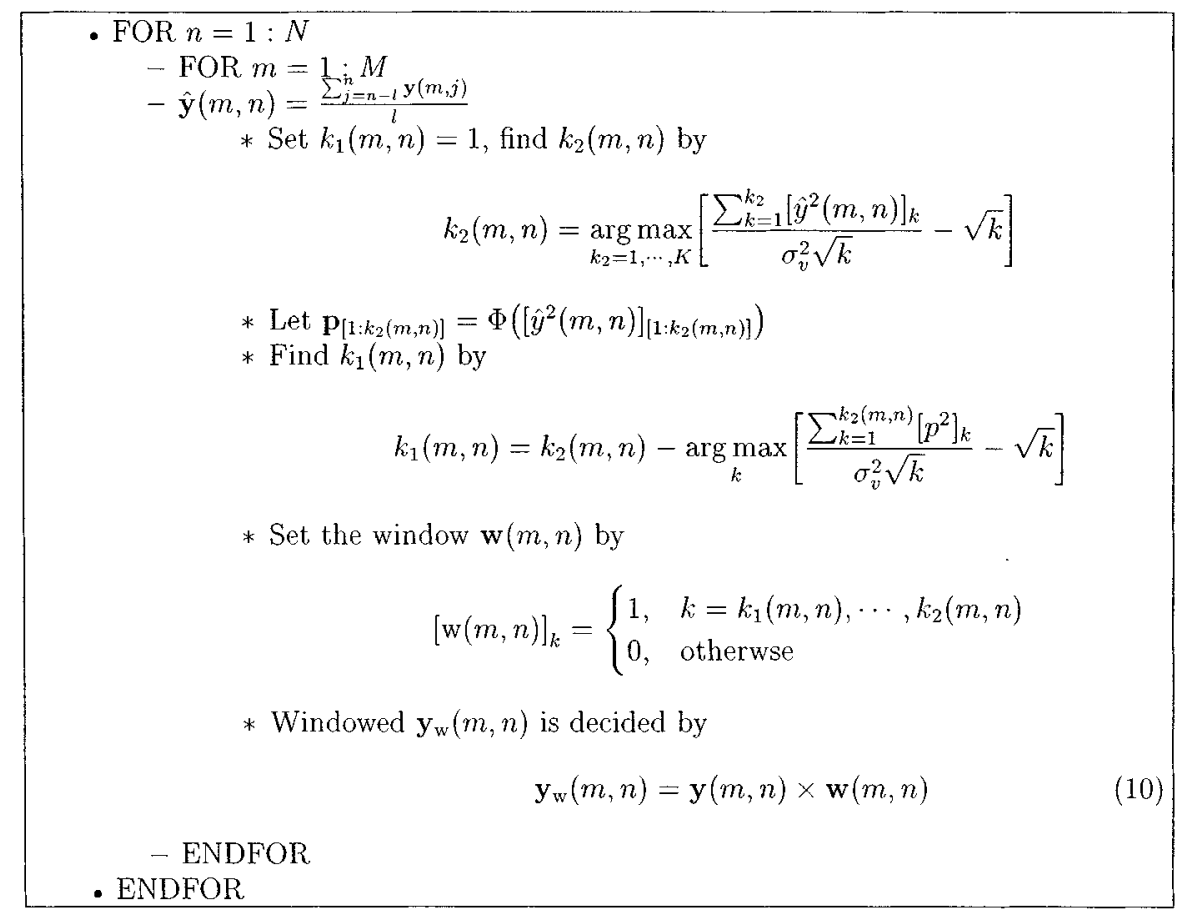

Fig. 5. Steps of deciding window $\mathbf{w}(m, n)$ and $\mathbf{y}_{\mathbf{w}}(m, n)$.

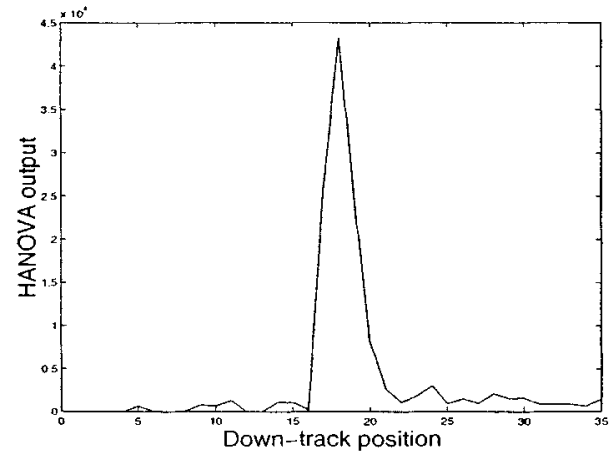

(a) HANOVA result $Y(n)$ over a metal mine.

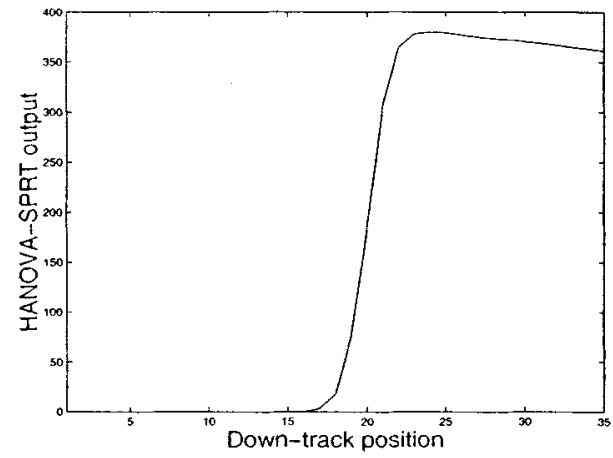

(b) Output $U(n)$ of SPRT following HANOVA

Fig. 6. HANOVA and SPRT processing results for metal mine data shown in Fig. 3. Unit in down-track position is about $7.6 \mathrm{~cm}$.

Note that $\mathbf{y}_{\mathrm{w}}(m, n)$ can be of different lengths because of different windows applied. Fig. 6(a) shows the result of applying HANOVA to the data in Fig. 3. Where the HANOVA output is high, so too is the likelihood of an object being present. Thus, in Fig. 6(a), the object is clearly detectable. More examples involving different types of objects will be given in Section III.

\section{B. Down-Track Processing}

While HANOVA detects statistical significance at one stop of the array, it does not capture the object signal structure seen as the array moves down-track. To improve detection performance, we employ a sequential detection scheme to process $Y(n)$ recursively as $n$ increases in order to identify the transient signal arising from the mine [4], [25]. Specification of this SPRT begins by noting that under our models $Y(n)$ takes on a $\chi^{2}$ distri- bution under both $H_{0}$ and $H_{1}$. Letting $\lambda=\sum_{m=1}^{M}\left(k_{2}(m, n)-\right.$ $\left.k_{1}(m, n)\right)$, standard statistical analysis [14] yields

$$
\begin{aligned}
& H_{0}: Y(n) \sim \chi_{\lambda}^{2}(0) \\
& H_{1}: Y(n) \sim \chi_{\lambda}^{2}\left(\delta^{2}(n)\right)
\end{aligned}
$$

for $n=1, \ldots, N$ where the notation $x \sim \chi_{p}^{2}\left(\delta^{2}\right)$ indicates that the random variable $x$ is distributed according to a $\chi^{2}$ law of order $p$ and noncentrality parameter $\delta^{2}$ [24]. For the GPR problem, it is easy to show that

$$
\delta^{2}(n)=\frac{1}{\sigma_{v}^{2}} \sum_{m=1}^{M}\|\mathbf{s}(m, n) \times \mathbf{w}(m, n)\|^{2} .
$$

For our problem, the length of each window is large (on the order of hundreds) and the central limit theorem permits us to 
- $U(1)=0$

- FOR $n=2, \cdots, N$

$-\mu_{1}(n)=Y(n)$

- Form $\sigma_{1}^{2}(n)$ according to $(16)$

- Form $u(n)$ according to $(15)$

$-U(n)=\max (0, U(n-1)+u(n))$

- ENDFOR

- IF $U(n)>\alpha$, declare object, set $U(n)=0$, ENDIF

Fig. 7. Sequential processing.

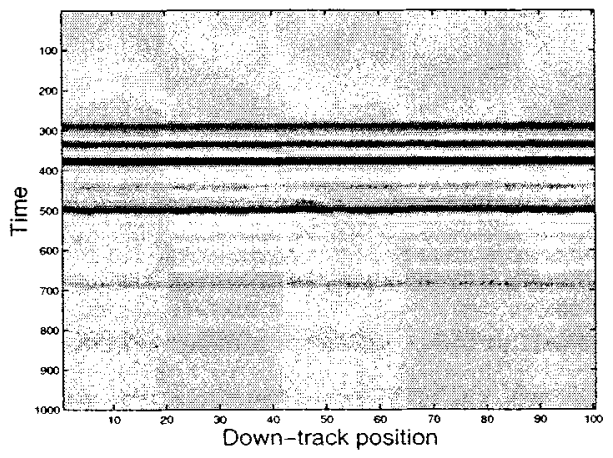

(a)

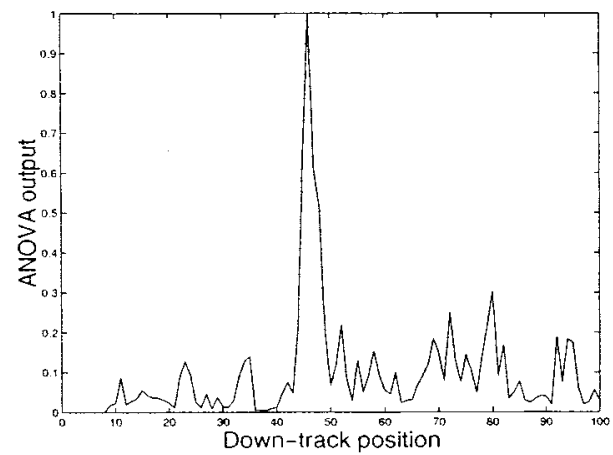

(c)

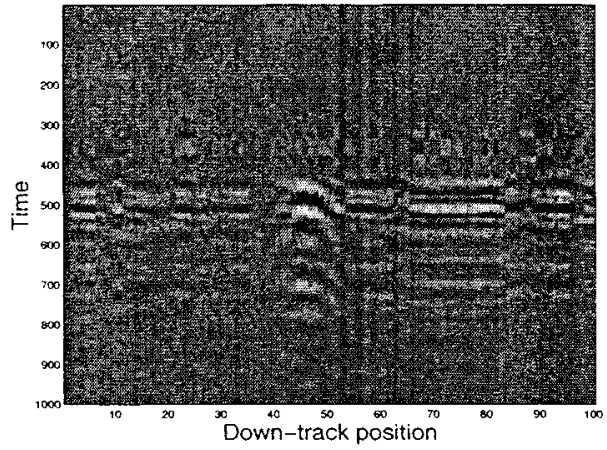

(b)

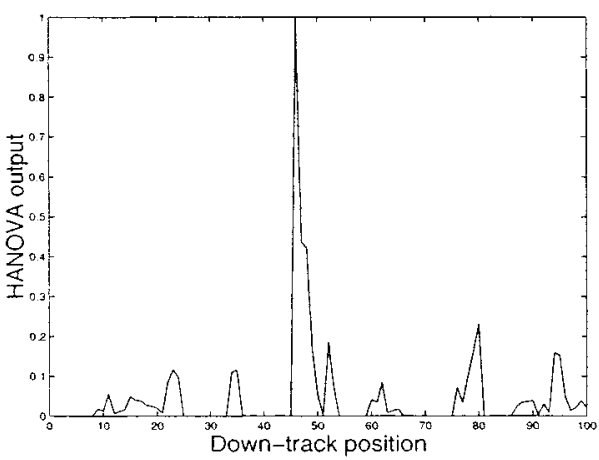

(d)

Fig. 8. Results of a single GPR measurement above a steel object around position 50. (a) Raw observation. (b) Observation after nominal background removal. (c) ANOVA output. (d) HANOVA output. In (a) and (b), unit in down-track position is about $7.6 \mathrm{~cm}$, unit in time axes is $0.02 \mathrm{~ns}$.

approximate the $\chi^{2}$ distribution using a Gaussian distribution [24]. We then have

$$
\begin{aligned}
H_{0}: Y(n) & \sim N\left(\mu_{0}, \sigma_{0}^{2}\right) \equiv N(\lambda, 2 \lambda) \\
H_{1}: Y(n) & \sim N\left(\mu_{1}(n), \sigma_{1}^{2}(n)\right) \\
& \equiv N\left(\lambda+\delta^{2}(n), 2 \lambda+4 \delta^{2}(n)\right) .
\end{aligned}
$$

At stop $n$, the log likelihood ratio for the hypothesis testing problem in (14) is

$$
u(n)=\ln \frac{p_{n}(Y(n))}{p_{0}(Y(n))}, \quad n=1, \ldots, N
$$

where $p_{n}(Y(n))$ is the probability density function (pdf) of $Y(n)$ evaluated at the $n$th stop under $H_{1}$ and $p_{0}(Y(n))$ is the pdf of $Y(n)$ evaluated under $H_{0}$. Under $H_{0}, \mu_{0}$ and $\sigma_{0}^{2}$ are estimated using data from an object-free area. Therefore, for this algorithm, the GPR array must start by collecting data in a calibra- tion region to initialize these variables. Under $H_{1}$, one difficulty with generating $u(n)$ is that $\mu_{1}(n)$ and $\sigma_{1}^{2}(n)$ are typically not known a priori since the underlying $\mathbf{s}(m, n)$ are not assumed known. It turns out that we only need to estimate $\mu_{1}(n)$, and $\sigma_{1}^{2}(n)$ can be found from the following relation:

$$
\begin{aligned}
\sigma_{1}^{2}(n) & =2 \lambda+4 \delta^{2}(n)=2 \lambda+4\left[\mu_{1}(n)-\lambda\right] \\
& =4 \mu_{1}(n)-2 \lambda .
\end{aligned}
$$

At the $n$th stop, we estimate the mean of $Y(n)$ by its maximumlikelihood estimator $\mu_{1}(n)=Y(n)$ [26].

The SPRT statistic $U(n)$ is a cumulative sum, changing with the acquisition of each new $u(n)$

$$
U(n)=\max (0, U(n-1)+u(n)) .
$$

Because subsurface object detection is a binary hypothesis testing problem, e.g., we are only interested in knowing whether 


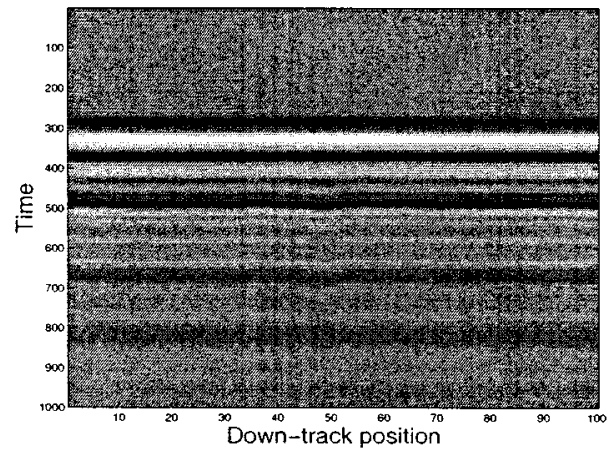

(a)

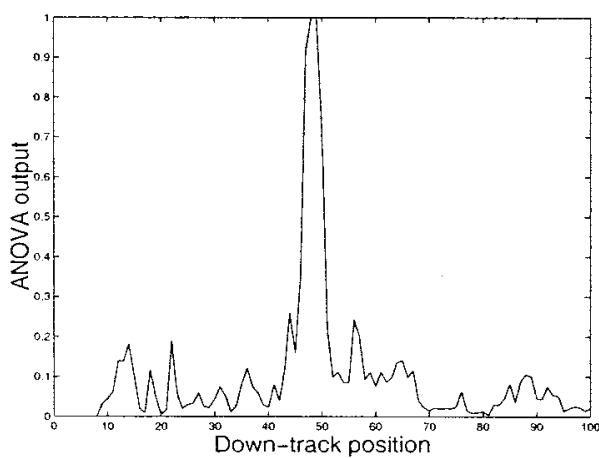

(c)

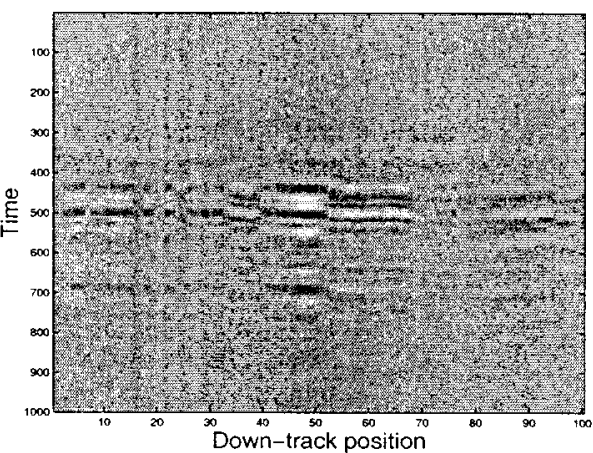

(b)

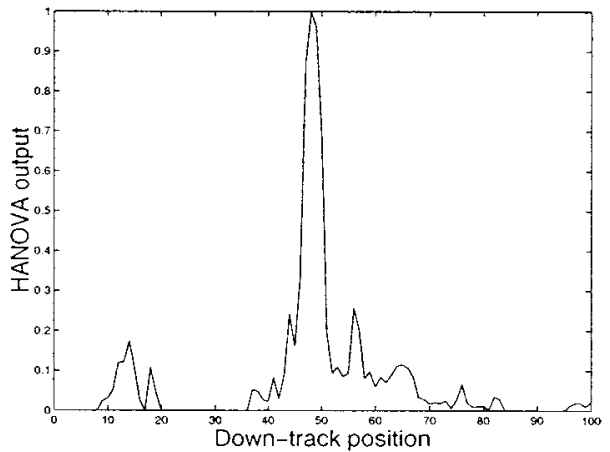

(d)

Fig. 9. Comparison between the ANOVA and HANOVA. (a) Raw data over an M19, anti-tank mine, buried at position 50. (b) Demeaned data. (c) Result of the ANOVA over the M19. (d) Result of the HANOVA. Unit in down-track position is about $7.6 \mathrm{~cm}$.

there is a buried object, the SPRT statistic is bounded from lower bound, zero. When $U(n-1)+u(n)$ is negative, $U(n)$ is reset to zero. For a preset threshold $\alpha$, the SPRT will make one of two decisions at each $n$

$$
\begin{aligned}
& U(n) \geq \alpha \Rightarrow \text { choose } H_{1} \\
& U(n)<\alpha \Rightarrow \text { take another observation. }
\end{aligned}
$$

The sequential detection is then essentially a repeated SPRT [5] and summarized in Fig. 7. Fig. 6(b) shows the sequential test statistic when the SPRT is applied to the data in Fig. 6(a). Because the SPRT in (17) has the form of a modified "integrator," a typical time series for the SPRT statistic takes a step-like form. The larger and sharper the step, the more likely it is that a target is present. At the position where there is an object, the sequential test statistic has a clear upward change again indicating the existence of an object at about position 16.

\section{EXAMPLES}

In this section we use field data as examples to illustrate the performance of our method. The field data are collected by both single GPR and GPR arrays at different test sites. For each data set, we compare the results from using standard ANOVA, HANOVA, ANOVA followed by SPRT, and HANOVA followed by SPRT. Comparison indicates that generally HANOVA performs better than ANOVA, and with SPRT, both ANOVA and HANOVA make fewer false alarms. In other words, HANOVA with SPRT gives the best receiver-operating characteristics (ROCs), as we shall see later in this section. Test sites are different in the number of targets buried underneath, moisture of the soil, roughness of the ground surface, and level of vegetation in the test sites.

At first, we apply our method on data collected by single GPR at Socorro test site, New Mexico. Data were collected by a GPR moving along a linear track at $5 \mathrm{~km} / \mathrm{h}$ sampling every $7.6 \mathrm{~cm}$. Some data are taken under relatively favorable condition, while most are from more hostile test sites which involve rough ground surface and other clutter. Fig. 8 compares results of ANOVA and HANOVA on a buried steel object at position 50. For comparison, the outputs of ANOVA and HANOVA are normalized to one. It is observable that while both methods detect the object easily, the HANOVA is better in suppressing noise output where there is no object, e.g., at position 1 through 40 and 60 through 100, Fig. 8(c) and (d). Fig. 9 shows the results from detecting a plastic mine, M19, at position 50. Again, the HANOVA performs better in suppressing noise. At positions 20 through 40, the HANOVA creates a much lower noise level than the ANOVA does. Similarly, the HANOVA produces a cleaner output at the end of the run. The order of MA process used in window selection is 4 .

Fig. 10 shows the results of ANOVA and HANOVA in detecting an anti-tank mine, TM62, from a very "noisy" data set. The mine is buried at position 60. Outputs of both HANOVA and 


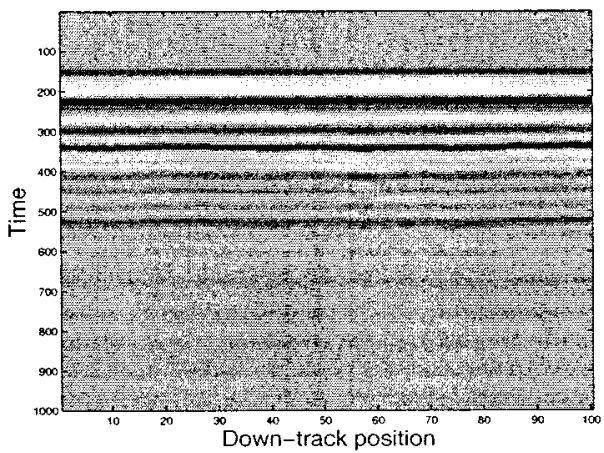

(a)

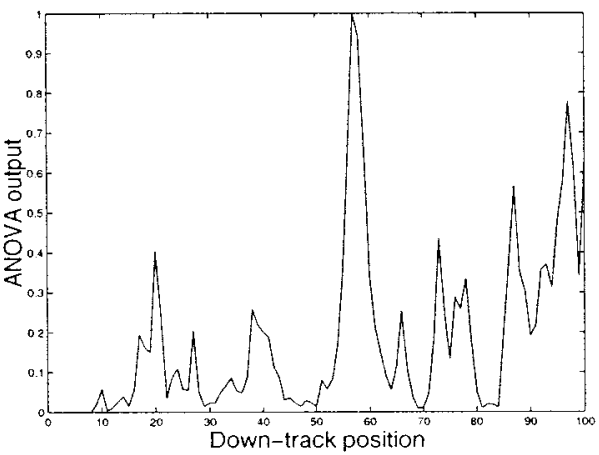

(c)

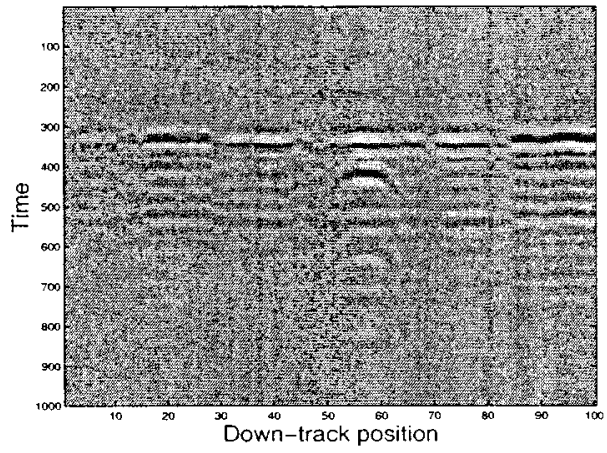

(b)

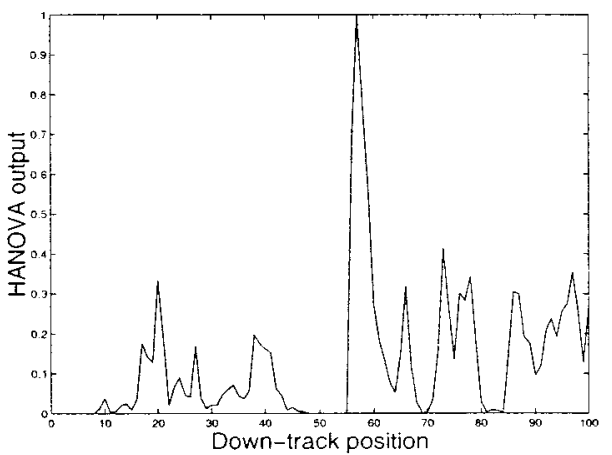

(d)

Fig. 10. More comparison between the ANOVA and HANOVA. (a) Raw data over a TM62, anti-tank mine, buried to the side of the track at position 58. (b) Demeaned data. (c) Result of the ANOVA. (d) Result of the HANOVA. Unit in down-track position is about $7.6 \mathrm{~cm}$.

ANOVA consist of the correct detection and some false alarms. The HANOVA maintains a better performance than the ANOVA in the sense that, for a given detection threshold, the HANOVA would generally have a smaller number of false alarms. For the HANOVA, no false alarm will be declared for a threshold greater than 0.5, while for the ANOVA, the threshold must be set above 0.8 to avoid making a wrong decision. Between threshold 0.5 and 0.8 , the ANOVA will make two false alarms while the HANOVA has zero false alarms.

Next, by comparing the outputs of the SPRT in the above three examples, we see that sequential processing generates fewer false alarms than by using ANOVA (or HANOVA) only (Fig. 11). In all three examples, SPRT following HANOVA performs better than SPRT following ANOVA, in the sense that the jump at the position of the buried object is sharper and the curve is close to zero-level in the object-free area.

To study the ROC of the method, we test our method on multiple runs of different types of targets. Fig. 12(a) shows the ROC curves of ANOVA and HANOVA to detect 60 metallic objects. The objects include metallic mines such as TM15, TM46, and PMN. Fig. 12(b) shows the ROC of ANOVA-SPRT and HANOVA-SPRT. Compared with Fig. 12(a), SPRT improves the performance of both ANOVA and HANOVA. In generating these curves, a correct identification of any of the objects was taken to be a "detection" whether or not the object itself was a mine. Indeed, as noted in the Introduction, the algorithm in this paper is intended only to detect the presence of objects below the array and not to solve the classification problem. Still, given the "real-world" conditions under which the data were taken, the low false-alarm rates here point to the robustness of our approach.

Next, we compare the performance of ANOVA, HANOVA, ANOVA-SPRT, and HANOVA-SPRT in detecting 70 plastic mines. The mines are M19, VS-1.6, T72, and C4A1. Fig. 13 shows the ROC curves of the above four methods. It is seen that both the ANOVA-SPRT and HANOVA-SPRT perform better than the ANOVA and HANOVA, respectively.

As another example, we test our method on a different array radar system at another test site. The setup of the GPR array is shown in Fig. 14. There is one transmitter in this system. In front of the transmitter, four receivers are positioned in a $2 \times 2$ pattern. Above the transmitter and the receivers there is a hyperbolic reflection plate; it is set so that the transmitter is at the focal point of the reflection plate. The array moves on a linear track to collect data. At each step, the transmitter sends a spherical wave to the reflection plate and after reflection, the sphere wave becomes plane wave. The four receivers then collect reflection of this plane wave from the ground. The system has the advantage of generating plane wave and points it forward to reduce ground reflection. The order of MA process used in window selection is 4. Fig. 15 displays collected data from the two front receivers at the Dedham test site of Northeastern University and 


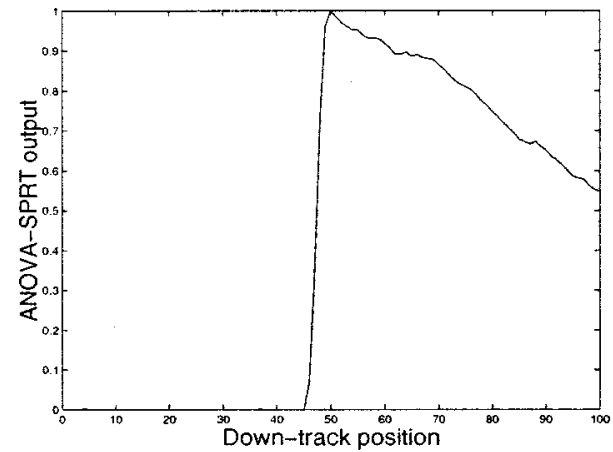

(a)

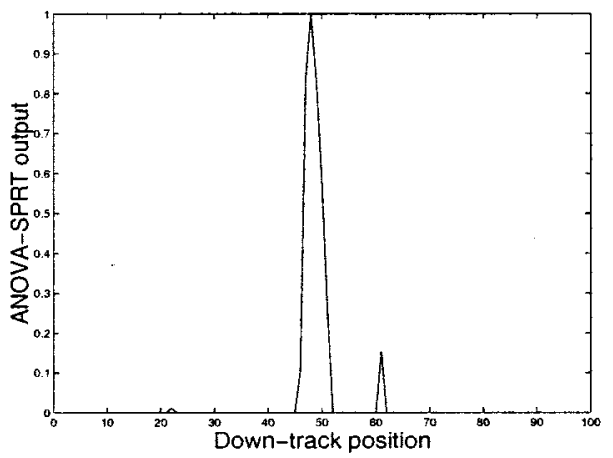

(c)

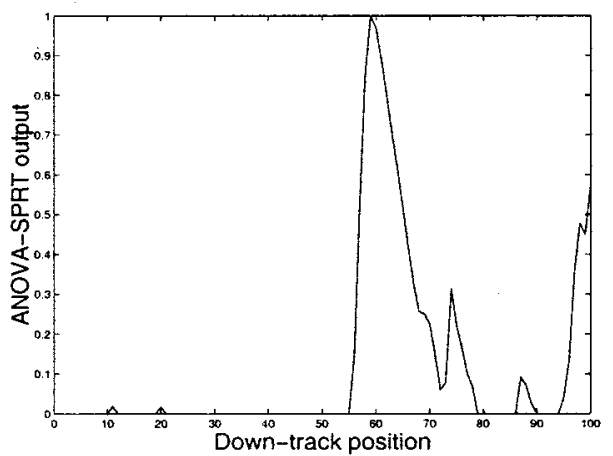

(e)

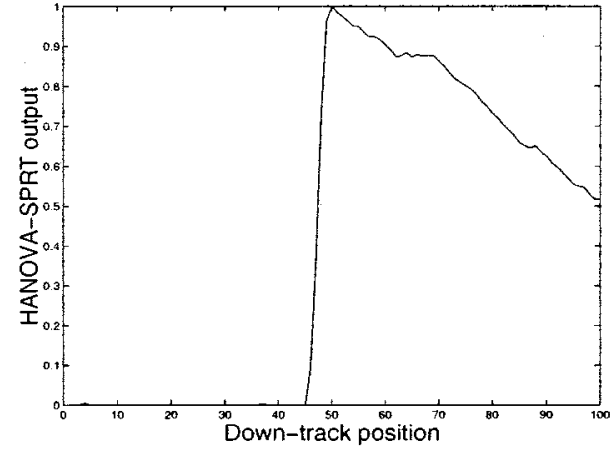

(b)

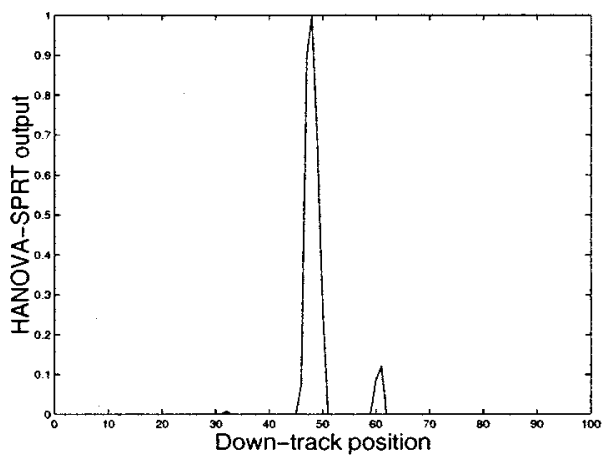

(d)

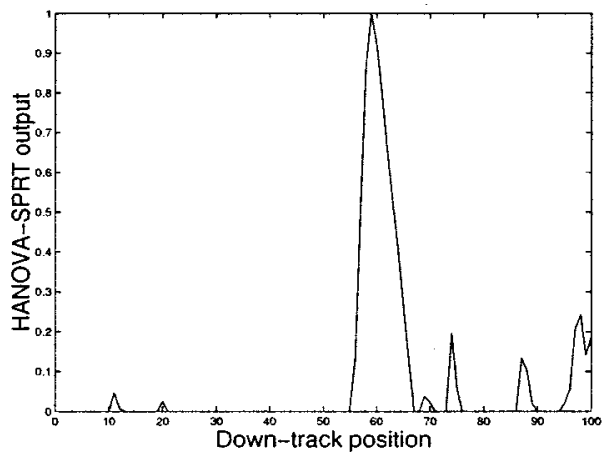

(f)

Fig. 11. Results of the SPRT, a buried steel object. (a) Output of ANOVA-SPRT. (b) Output of HANOVA-SPRT; a buried M19. (c) Output of ANOVA-SPRT. (d) Output of HANOVA-SPRT; a buried TM62. (e) Output of ANOVA-SPRT. (f) Output of HANOVA-SPRT. Unit in down-track position is about $7.6 \mathrm{~cm}$.

the corresponding signal after background removal. In an area of $58 \mathrm{~m}^{2}$, there are 12 buried landmines of different types, such as M19, PMN, VS-2.2, and so on. Using our method we are able to detect all 12 mines with a few false alarms (see Fig. 16). The results are similar to those obtained by a single GPR. For a detection rate above $90 \%$, the HANOVA has a significantly smaller number of false alarms.

\section{SUMMARY}

In this paper, we have proposed a sequential, high-dimensional ANOVA to process GPR returns. The method is tested on real data and has a relaxed requirement on the physical model used in the processing routine. The method is on-line implementable and has a linear computational load. The method works in two stages: first it looks for statistically significant difference from array observations, and second, it applies a sequential detection as new data are obtained. HANOVA is powerful in the sense of maximizing probability of detecting statistically significant difference among subdimensions of a full vector of observations. Sequential detection recursively processes the result of the HANOVA and enables real-time processing as new data are collected. We have demonstrated the performance of this technique on samples of field data. 


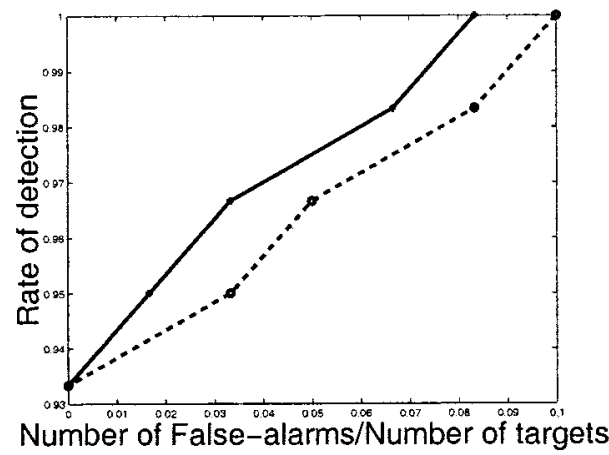

(a)

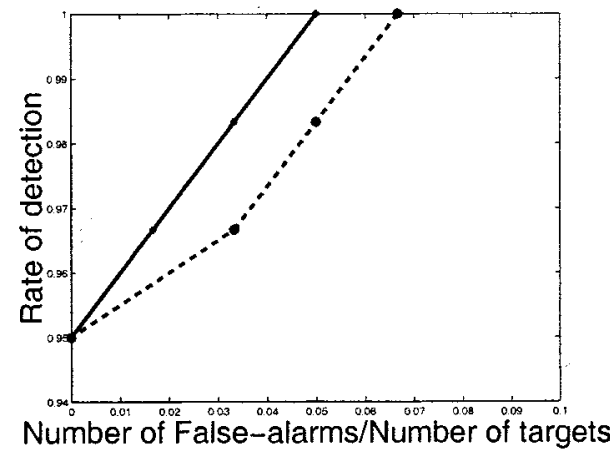

(b)

Fig. 12. Rate of detection and rate of false alarm in detecting metallic objects. Solid line is the result of HANOVA, and the dashed line is the result of ANOVA. (a) ANOVA versus HANOVA. (b) ANOVA-SPRT versus HANOVA-SPRT.

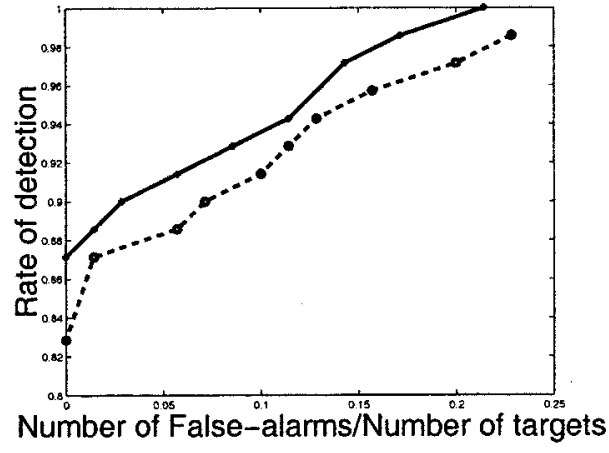

(a)

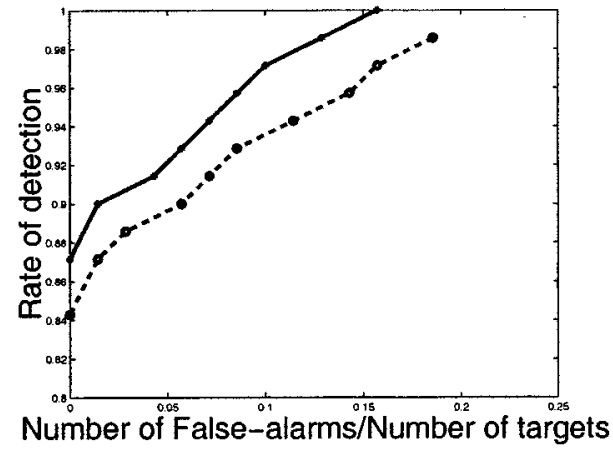

(b)

Fig. 13. Rate of detection and rate of false alarm in detecting plastic mines. The solid line is the result of HANOVA, and the dashed line is the result of ANOVA. (a) ANOVA versus HANOVA. (b) ANOVA-SPRT versus HANOVA-SPRT.
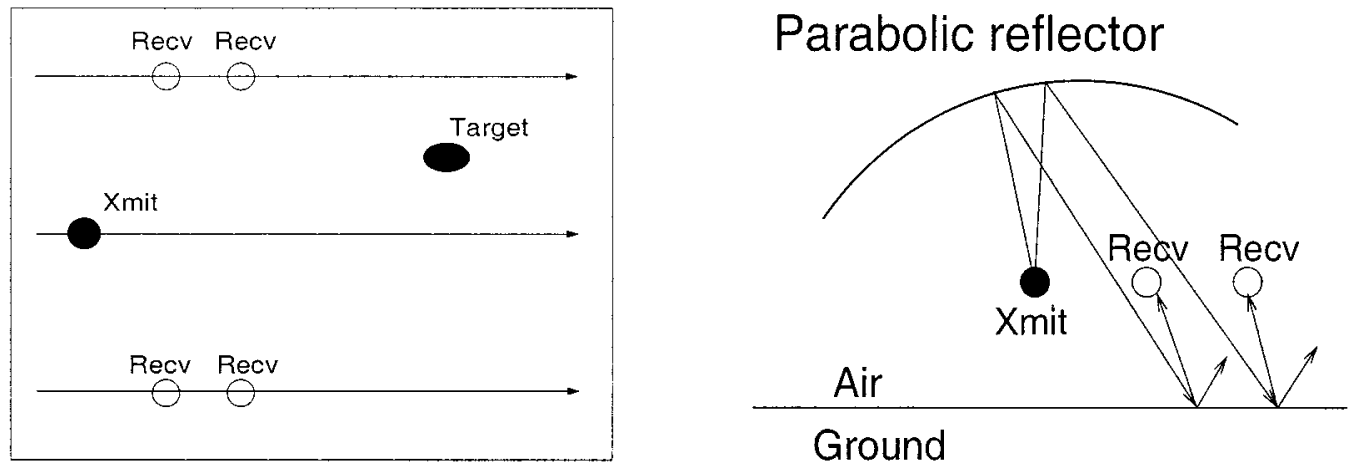

(a)

Fig. 14. GPR array used at Dedham test site of Northeastern University. (a) Plane view. (b) Side view.

Future research will focus on localization. Localization is based on optimized frequency-wavenumber (F-K) migration. $\mathrm{F}-\mathrm{K}$ migration is an inversion method that back-propagates the wavefield from the ground surface to the subsurface and constructs an image of subsurface reflectivity. Regular F-K migra- tion is well modeled for seismic signal processing. Though GPR signal is different from seismic signal, F-K migration can still work very well in processing GPR signals. Nonetheless, improvement in terms of resolution and accuracy can be achieved by considering optimization in F-K migration. 


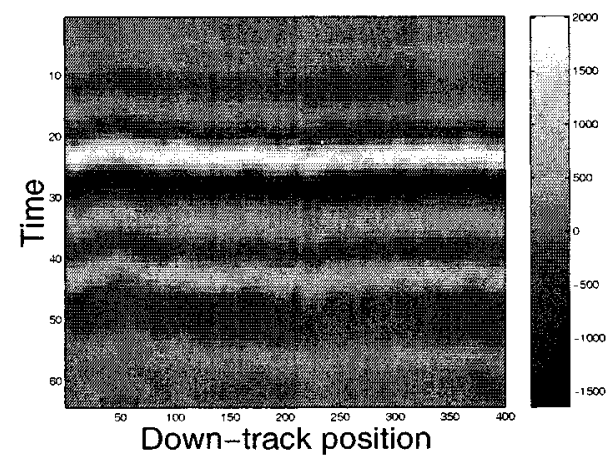

(a)

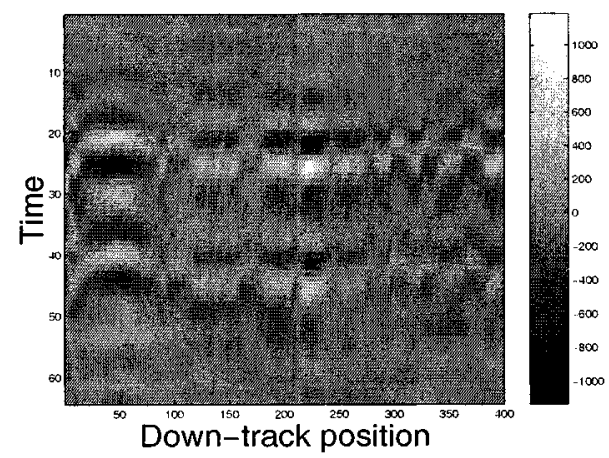

(c)

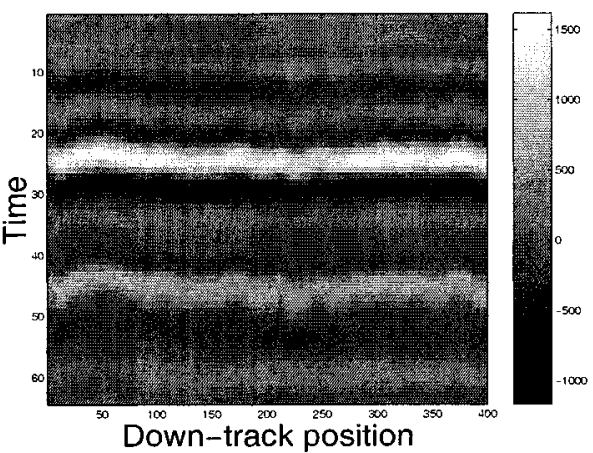

(b)

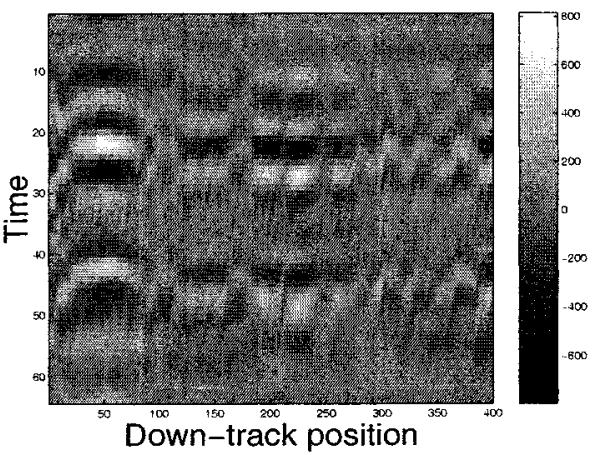

(d)

Fig. 15. GPR data from the Dedham test site (a) from the left front receiver, (b) from the right front receiver, (c) signal (a) after background removal, and (d) signal of (b) after background removal. Unit in down-track position is about $7.6 \mathrm{~cm}$. Unit in time axes is $120 \mathrm{ps}$.

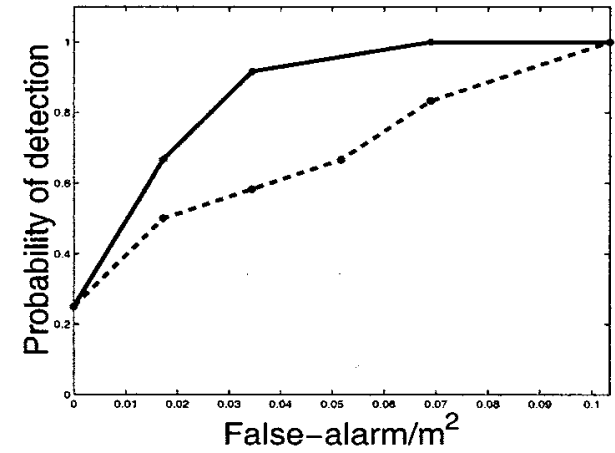

Fig. 16. ROCs of the Dedham test. Solid line: HANOVA-SPRT; dashed line: ANOVA-SPRT.

\section{APPENDIX}

\section{WINDOW SELECTION IN HANOVA}

Ideally, we want to find a window that is sensitive to the presence of a signal and provides little response in the test statistic when there is noise only. But these two requirements are often in conflict with each other. From (8), we can change the order $l$ of the MA process to control the window we use. The smaller $l$ is, the more sensitive the window is to the presence of signal and strong noise. On the other hand, the larger $l$ is, the more robust will the statistic be to noise, which translates into a smaller probability of false alarm. But a large $l$ reduces sensitivity of the HANOVA to signal. Fig. 17 shows the effect of $l$ on window selection and the corresponding HANOVA results. Three different $l$ are used, i.e., $l=1,4,9$. In the data, there are three mine objects: two metal mines at positions 110 and 170 and a weak mine object at position 25 . For comparison, we normalize the HANOVA outputs in each case by its maximum value, which corresponds to the strong metal mine buried at position 110 . Fig. 17(a) and (b) show the window chosen by an MA of order 1 and the resulting HANOVA output. The two strong objects can be detected at a threshold of 0.7 , the weak object can only be found at a threshold of 0.2. Fig. 17(c) shows the window chosen by an MA of order 4 . The window oscillates much less than the window in Fig. 17(a). From the HANOVA result, Fig. 17(d), we can find all the three objects at a threshold of 0.3. Increasing the order of MA process can make the results worse, Fig. 17(e) and (f). A large window reduces the sensitivity of the HANOVA to the signal and actually makes detection more difficult. Now the weak object at position 25 can not be detected at a threshold greater than 0.3. As a guideline, we find that MA processes of order between 3 and 10 yield good windows both in sensitivity to signal and robustness to noise. This selection is affected by the step-size of the array. An array moving at small step-size will allow an MA process of large $l$ in selecting windows, and vice versa. 


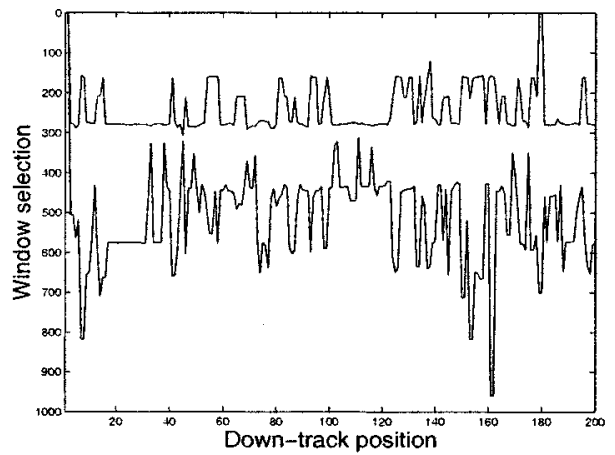

(a)

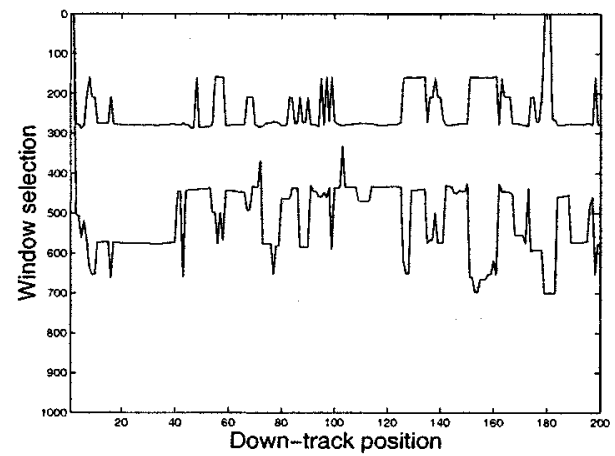

(c)

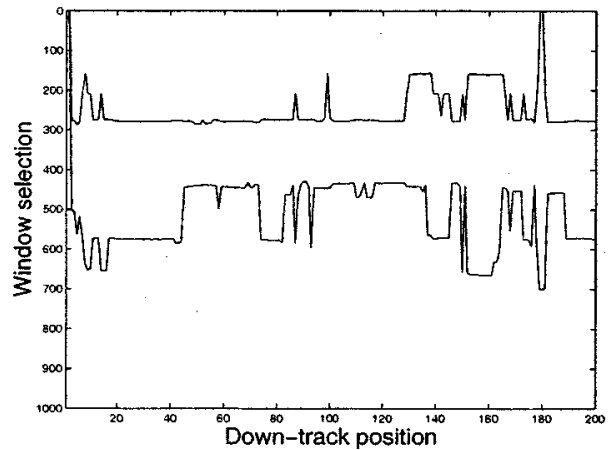

(e)

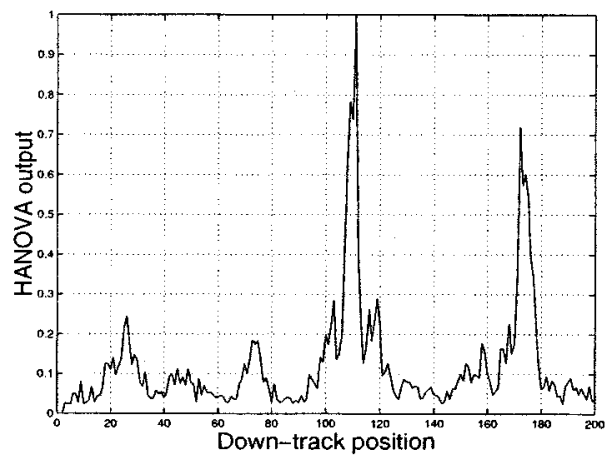

(b)

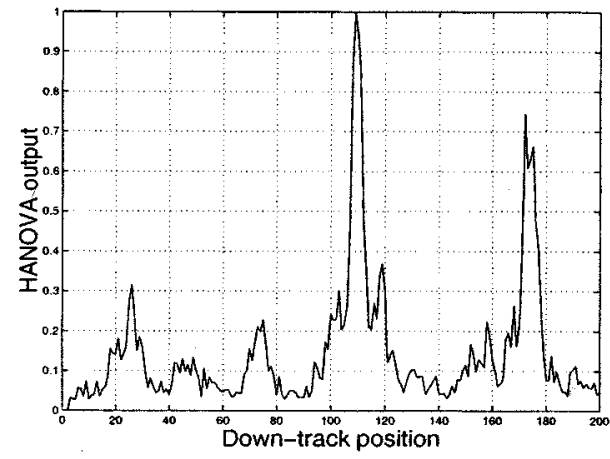

(d)

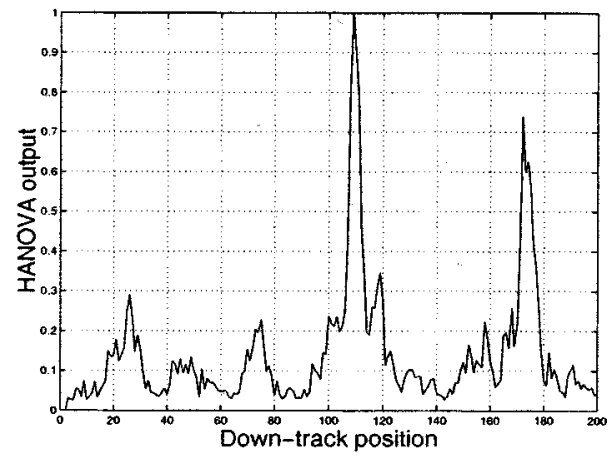

(f)

Fig. 17. Choices of window and effect on HANOVA. The lower line is $k_{1}(m, n)$, and the upper line is $k_{2}(m, n)$. (a) Window selected by an order-1 MA process. (b) HANOVA result from the window to the left. (c) Window selected by an order-4 MA process. (d) HANOVA result from the window to the left. (e) Window selected by an order-10 MA process. (f) HANOVA test result from the window to the left. Unit in down-track position is about $7.6 \mathrm{~cm}$.

\section{ACKNOWLEDGMENT}

The authors would like to acknowledge the very helpful comments from anonymous reviewers whose advice has improved the quality of this paper. They are thankful to ARO MURI for the suppport. They would also like to thank the Center for Subsurface Sensing and Imaging Systems for this support and help.

\section{REFERENCES}

[1] L. Peter, Jr., J. J. Daniels, and J. D. Young, "Ground penetrating radar as a subsurface environmental sensing tool," Proc. IEEE, vol. 82, pp. 1802-1822, Dec. 1994.
[2] C. M. Rappaport, S. G. Azevedo, T. Rosenbury, and J. Gough, "Handheld forward-looking focused array mine detection with plane wave excitation," in Proc. SPIE, vol. 4038, AeroSense Detection and Remediation Technologies for Mines and Minelike Targets V, 2000, pp. 1118-1126.

[3] J. Kositsky and P. Milanfar, "A forward-looking high-resolution GPR system," in Proc. SPIE, vol. 3710, AeroSense Detection and Remediation Technologies for Mines and Minelike Targets IV, 1999, pp. 1052-1062.

[4] C. W. Helstrom, Elements of Signal Detection and Estimation. Englewood Cliffs, NJ: Prentice-Hall, 1995.

[5] M. Basseville and I. V. Nikiforov, Detection of Abrupt Changes: Theory and Application. Englewood Cliffs, NJ: Prentice-Hall, 1993. 
[6] J. M. Bourgeois and G. S. Smith, "A fully three-dimensional simulation of a ground-penetrating radar: FDTD theory compared with experiment," IEEE Trans. Geosci. Remote Sensing, vol. 34, pp. 36-44, Jan. 1996.

[7] J. R. Wait, Geo-Electromagnetism. New York: Academic, 1982.

[8] G. R. Olhoeft, "Application of ground penetrating radar," in Proc. GPR'96, Sendai, Japan, Sept. 1996, pp. 1-3.

[9] K. Demarest, Z. Huang, and R. Plumb, "An FDTD near- to far-zone transformation for scatterers buried in stratified ground," IEEE Trans. Antennas Propagat., vol. 44, pp. 1150-1157, Aug. 1996.

[10] P. G. Gader, H. Hichem, B. N. Nelson, G. Vaillette, and J. M. Keller, "New results in fuzzy set based detection of landmines with GPR," in Proc. SPIE, vol. 3710, AeroSense Detection and Remediation Technologies for Mines and Minelike Targets IV, 1999, pp. 1075-1084.

[11] M. R. Bradley, T. R. Witten, R. McCummins, M. Crowe, S. Stewart, and M. Duncan, "Mine detection with a multichannel stepped-frequency ground-penetrating radar," in Proc. SPIE, vol. 3710, AeroSense Detection and Remediation Technologies for Mines and Minelike Targets IV, 1999, pp. 953-960.

[12] T. Dogaru and L. Carin, "Time-domain sensing of targets buried under a rough air-ground interface," IEEE Trans. Antennas Propagat., vol. 46, pp. 360-372, Mar. 1998

[13] UXO signature data (1999). [Online]. Available: http://www.denix.osd. mil/denix/Public/News/UXOCOE/Sigdata/sigdata.html

[14] J. Fan, "Test of significance based on wavelet thresholding and Neyman's truncation," J. Amer. Stat. Assoc., vol. 91, pp. 674-688, 1996.

[15] J. Fan and S. Lin, "Test of significance when data are curves," J. Amer. Stat. Assoc., vol. 93, pp. 1007-1021, 1998

[16] P. Shi and E. L. Miller, "Baseband Wiener filter processing for mine detection from scanned laser induced acoustic data," in Proc. SPIE, vol. 3710, AeroSense Detection and Remediation Technologies for Mines and Minelike Targets IV, 1999, pp. 1373-1384.

[17] X. Xu, E. L. Miller, and C. M. Rappaport, "Combined high-dimensional analysis of variance (HANOVA) and sequential probability ratio test (SPRT) to detect buried mines," Proc. SPIE, vol. 4038, pp. 1037-1046, Apr. 2000.

[18] K. C. Ho and P. D. Gader, "Correlation based landmine detection using GPR," Proc. SPIE, AeroSense Detection and Remediation Technologies for Mines and Minelike Targets V, vol. SPIE 4038, pp. 1088-1095, 2000.

[19] X. Xu, E. L. Miller, and C. M. Rappaport, "Statistically-based sequential detection of buried mines from array ground penetrating radar data," Proc. SPIE AeroSense Detection and Remediation Technologies for Mines and Minelike Targets IV, vol. SPIE 3710, pp. 1063-1075, 1999.

[20] M. El-Shenawee and C. M. Rappaport, "Quantifying the effects of different rough surface statistics for mine detection using the FDTD technique," Proc. SPIE, vol. 4038, pp. 966-975, 2000.

[21] L. Collins, S. Tantum, P. Gao, J. Moulton, L. Makowsky, D. Reidy, and D. Weaver, "Improving detection of low-metallic content landmines using EMI data," Proc. SPIE, vol. 4038, pp. 14-24, 2000.

[22] J. W. Brooks, L. van Kempen, and H. Sahli, "A primary study in adaptive clutter reduction and buried minelike target enhancement from GPR data," Proc. SPIE, vol. 4038, pp. 1183-1192, 2000.

[23] B. K. Ghosh, Sequential Tests of Statistical Hypotheses. Reading, MA: Addison-Wesley, 1970.

[24] S. C. Port, Theoretical Probability for Applications. New York: Wiley, 1994.

[25] D. Siegmund, Sequential Analysis: Tests and Confidence Intervals. New York: Springer-Verlag, 1985.

[26] S. M. Kay, Fundamentals of Statistical Signal Processing: Estimation Theory. Englewood Cliffs, NJ: Prentice-Hall, 1993.
Xiaoyin Xu, photograph and biography not available at the time of publication.

Eric L. Miller (S'90-M'95) received the S.B. S.M., and Ph.D. degrees, all in electrical engineering and computer science, from the Massachusetts Institute of Technology, Cambridge, in 1990, 1992, and 1994, respectively.

$\mathrm{He}$ is currently an Associate Professor in the Department of Electrical and Computer Engineering at Northeastern University, Boston, MA. His research interests include the use of multiscale and statistical methods for the solution of inverse problems in general and inverse scattering problems in particular and the development of computationally efficient, physically-based models for use in applications such as mine detection, target recognition, medical imaging, and environmental monitoring and remediation.

Dr. Miller is a member of Tau Beta Pi, Eta Kappa Nu, and Phi Beta Kappa. He received the CAREER Award from the National Science Foundation in 1996. $\mathrm{He}$ is currently serving as an Associate Editor for the IEEE TRANSACTIONS ON IMAGE PROCESSING.

Carey M. Rappaport (SM'96) received the S.B. degree in mathematics, the S.B., S.M., and E.E. degrees in electrical engineering in 1982, and the Ph.D. degree in electrical engineering in 1987, all from the Massachusetts Institute of Technology (MIT), Cambridge.

He has worked as a teaching and research assistant at MIT from 1981 until 1987, and during the summers at COMSAT Labs in Clarksburg, MD, and The Aerospace Corporation, El Segundo, CA. He joined the faculty at Northeastern University in Boston, MA, in 1987. He has been Professor of Electrical and Computer Engineering since July 2000. During Fall 1995, he was Visiting Professor of Electrical Engineering at the Electromagnetics Institute of the Technical University of Denmark, Lyngby, as part of the W. Fulbright International Scholar Program. He has consulted for Geo-Centers, Inc., PPG, Inc., and several municipalities on wave propagation and modeling, and microwave heating and safety. He is Principal Investigator of an ARO-sponsored Multidisciplinary University Research Initiative on Demining and Co-Principal Investigator of the NSF sponsored Center for Subsurface Sensing and Imaging Systems (CenSSIS) Engineering Research Center. He has authored over 175 technical journal and conference papers in the areas of microwave antenna design, electromagnetic scattering computation, and bioelectromagnetics, and has received two reflector antenna patents, two biomedical device patents and three subsurface sensing device patents.

Prof. Rappaport was awarded the IEEE Antenna and Propagation Society's H.A. Wheeler Award for best applications paper of 1985 . He is a member of Sigma Xi and Eta Kappa Nu.

Gary D. Sower received the Ph.D. degree in atmospheric physics from the New Mexico Institute of Mining and Technology, Socorro, in 1973.

In 1973, he joined the Albuquerque Division of EG\&G, now EG\&G Technical Services, Inc., where he is Principal Scientific Specialist. He has done extensive work in the design and construction of EMP (electromagnetic pulse) sensors and simulators, and was honored as an EMP Fellow in 1999. He has performed the design of metal detector and ground-penetrating radar subsystems for vehicle-mounted land mine detection systems, as well as developing feature extraction and target identification algorithms for the subsystems. He has published numerous papers on the above subjects. 